Arch. Tierz., Dummerstorf 50 (2007) 6, 628-629

Animal Genomics \& Bioinformatics Division, National Institute of Animal Science, Suwon 441-701 Korea

HOYOUNG CHUNG, SUNGHO YOON, MYUNGGI BECK, HEEJIN PARK, and JAEYOUNG KIM

\title{
Phylogenic analysis of the SLA-1 gene variants observed from Korean Jeju pig (Brief Report)
}

(Phylogenetische Analyse von SLA-1 Genvarianten beim koreanischen Jeju Schwein)

Background: The Swine Leukocyte Antigen (SLA) class I consists of SLA-1, SLA-2, and SLA-3 genes (CHARON et al., 2000) that are expressed on the surface of most nucleated cells. Pig organs mainly from miniature pigs have been used in studies on xenotransplantation (SMITH et al., 2005). It is important to understand genetic variations of the SLA-1 gene considering the SLA-mediated immune responses. Collecting information on single nucleotide polymorphisms (SNPs) and alleles of SLA-1 and their phylogenic relationships is important regarding future uses of the Korean Jeju Pig (KJP) in xenotransplantation.

\section{Procedure}

Primer sequences:

Primers were designed from the swine coding sequence (GenBank accession No.: AY247766 and AF464016) of porcine SLA-1 consisting of 8 exons in 1,087 bp length. forward primer: $\quad$ 5'-GGGCGTCGACCAGACTCCGAGGCTGAGG-3' (nt1-28) reverse primer: $\quad$ 5'-GAAGGTTCTCAATCCTTCCATTT-3' (nt1,544-1,566)

Identification of SLA-1 alleles:

Spleen tissues were collected from 23 individuals of the breed Korean Jeju Pig at an age of 30 weeks at National Institute of Animal Science, and total RNA was extracted. First strand cDNA was synthesized from pooled spleen tissues by random primers with Reverse Transcriptase. To amplify cDNAs SLA-1 specific primers were used with 2.5 $\mu \mathrm{l}$ of $10 \mathrm{X}$ reaction buffer, $10 \mu \mathrm{M}$ dNTP, $1 \mu \mathrm{l}$ of each primer ( $10 \mathrm{pM}), 50 \mathrm{ng}$ of cDNA, and 2 units of Taq DNA polymerase in a final volume of $25 \mu \mathrm{l}$. After heating at $95^{\circ} \mathrm{C}$ for $2 \mathrm{~min}$, a total of 35 cycles was adapted for denaturation at $94^{\circ} \mathrm{C} / 1 \mathrm{~min}$, annealing at $57^{\circ} \mathrm{C} / 1 \mathrm{~min}$, and polymerization at $72^{\circ} \mathrm{C} / 2 \mathrm{~min}$. The PCR products were inserted into pGEM T easy vector for blue and white selection with DH10B competent cells. The selected clones were sequenced.

Results: A total of 120 clones sized approximate 1,500 bp were produced. Finally, 46 clones were clustered with the Seqman program of DNAstar version 6.1 revealing 35 SNPs. Sequences of polymorphic KJP clones were submitted to GenBank (GenBank accession No.: DQ992486-92). The alignments contained several SNP positions, and especially, there were several nucleotides at positions $165(\mathrm{~A} / \mathrm{C} / \mathrm{T}), 166(\mathrm{~A} / \mathrm{C} / \mathrm{T})$, and 251 (G/C/A) based on the DQ992492 representing a consensus sequence of KJP. A phylogenic tree was constructed with DNAdist option (DNA distance matrix, Bio-edit version 7.0.1) for identifying a neighbor-joining phylogenic relationship. The identified consensus sequences (DQ992492) were submitted to the MHC Immuno Polymorphism Database (IPD) of the Major histocompatibility complex (MHC) with a 
new accession allele number SLA- $1 *$ w12hy01. The complete cDNA sequences of SLA-1 allele representing major alleles from NCBI have been aligned with the consensus sequences of KJP. As shown in the Figure, the KJP was closely clustered with the SLA-1*w12-w12Lw01 allele (Large White breed). This study provides new information on the genetic characteristics of KJP and phylogenic MHC diversity.

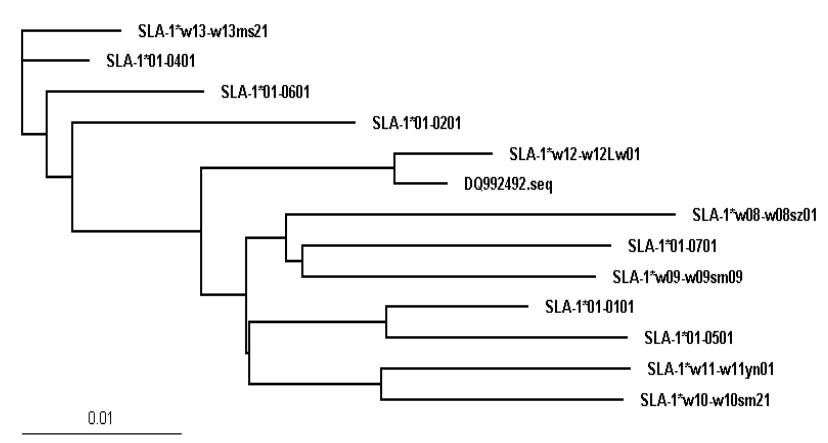

Figure: Phylogentic relationship among 13 SLA-1 alleles (Phylogenetische Beziehung von 13 SLA-1 Allelen) The GenBank accession No. of the SLA-1 alleles: AF464045 (SLA-1*01 0101), AF100665 (SLA-1*01 0201), AF014005 (SLA-1*01 0401), AF464044 (SLA-1*01 0501), AY135593 (SLA-1*01 0601), AF464036 (SLA1*01 0701), AF464013 (SLA-1*01 w08sz01), AY135594 (SLA-1*01 w09sm09), AY135589 (SLA-1*01 w10sm21), AY102469 (SLA-1*01 w11yn01), AY247766 (SLA-1*01 w12Lw01), AY459297( SLA-1*01 w13ms21).

Acknowledgements: This work was supported by Rural Development Administration in Korea for Production of Bio-Organs Research and Development Programs. This experiment was a contributing Regional Project 20070401034029 “Construction of BAC library from minipig and sequence analysis of SLA class I, II region in pig.

\section{References}

CHARON, P.; RENARD, C.; GAILLARD, C. R.; VAIMAN, M.:

The porcine major histocompatibility complex and related paralogous regions: a review. Genet. Sel. Evol. 32 (2000), 109-128

SMITH, D. M.; MARTENS, G. W.; HO, C. S.; ASBURY, J. M.:

DNA sequencing based typing of swine leukocyte antigens in Yucatan miniature pigs. Xenotransplantation 12 (2005), 481-488 